\section{Extrahepatic bile duct duplication complicated by choledocholithiasis}
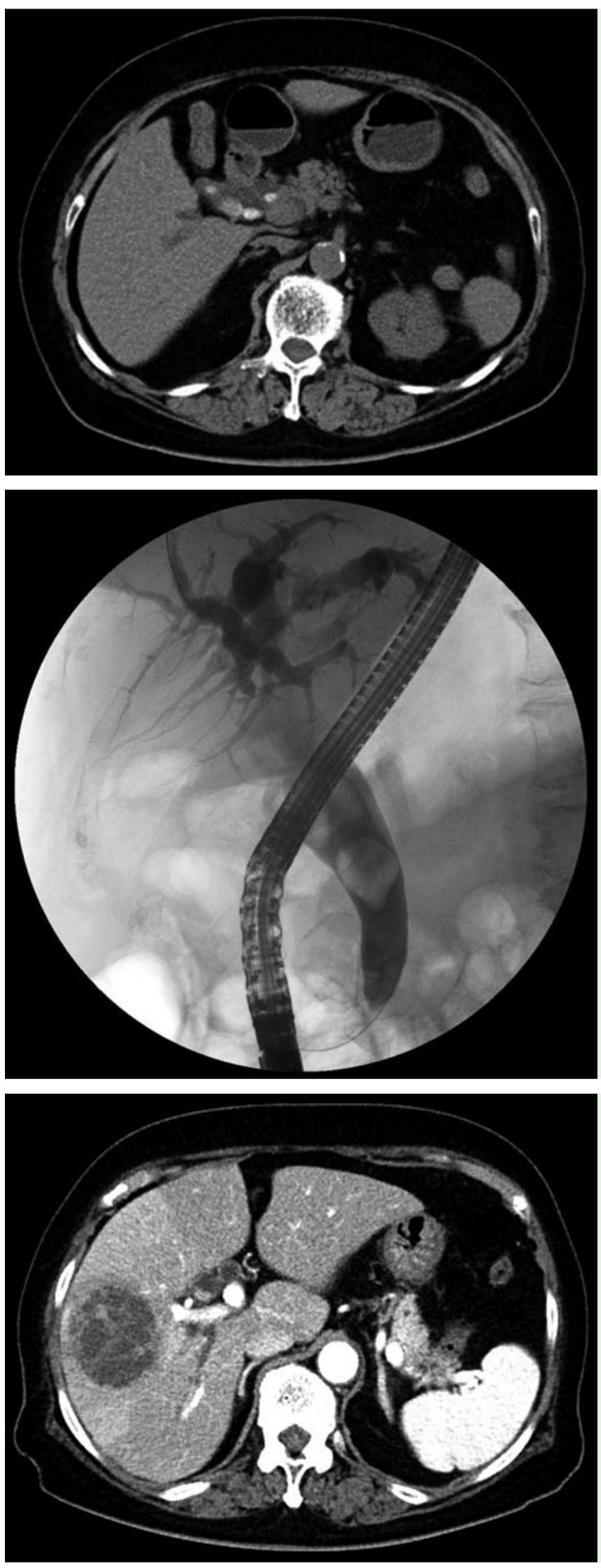

Fig. 1 Contrastenhanced computed tomography (CT) abdominal scan in a 77-year-old woman with epigastric and right upper quadrant abdominal pain demonstrated multiple stones together with diffuse biliary tree dilatation.

Fig. 2 Endoscopic retrograde cholangiopancreatography (ERCP) showed a markedly dilated extrahepatic bile duct with multiple stones in the biliary tree.

Fig. 3 Contrastenhanced abdominal CT 3 months after removal of the stones shown in $\bullet$ Fig. 2 demonstrated a multiseptated, low-density lesion in the right lobe of the liver, suggestive of abscess formation and bile duct stones in the extrahepatic bile duct.
A 77-year-old woman attended our hospital with a 10-day history of epigastric and right upper quadrant abdominal pain. Her past medical history included diabetes mellitus and hypertension, and she had undergone open cholecystectomy for gallstones 10 years previously. Physical examination revealed tenderness in the right upper quadrant of the abdomen. Laboratory findings were as follows: white blood cell count $4802 / \mu \mathrm{L}$, total bilirubin $0.58 \mathrm{mg} / \mathrm{dL}, \quad \gamma$-glutamyltransferase $17 \mathrm{IU} / \mathrm{L}$, and alkaline phosphatase $207 \mathrm{IU} / \mathrm{L}$. Abdominal computed tomography (CT) showed multiple stones with diffuse dilatation of the intrahepatic and extrahepatic biliary tree ( $\bullet$ Fig. 1 ). Endoscopic retrograde cholangiopancreatography (ERCP) showed a markedly dilated extrahepatic bile duct with multiple bile duct stones ( Fig.2). After endoscopic sphincterotomy followed by endoscopic papillary large balloon dilation, bile duct stones were removed using a Dormia basket and retrieval balloon, after which endoscopic retrograde biliary drainage was placed. At 3 months after discharge, the patient returned to our emergency department with fever and abdominal pain. Abdominal CT on this occasion showed a multiseptated, low-density lesion in the right lobe of the liver, about $6 \mathrm{~cm}$ in size, suggestive of abscess formation, and stone remnants in the biliary tree ( Fig.3). Percutaneous drainage of the hepatic abscess was performed. Magnetic resonance cholangiopancreatography showed extrahepatic bile duct duplication with choledocholithiasis. ERCP confirmed the bile duct duplication complicated by common bile duct stones, and the stone remnants in the right extrahepatic bile duct were removed ( $\bullet$ Fig. 4 ). Congenital extrahepatic bile duct duplication is extremely rare, and to the best of our knowledge there have only been two cases [1,2] of type Va extrahepatic bile duct duplication according to the modified classification of Choi et al. [1]. We report a case of extrahepatic bile duct duplication associated with multiple bile duct stones without a communicating channel between the two extrahepatic bile ducts.

Endoscopy_UCTN_Code_CCL_1AZ_2AK

Competing interests: None 




Fig. 4 ERCP demonstrated extrahepatic bile duct duplication without communication between the two separate bile ducts.

\section{Jay Song, Sung Bum Kim, Tae Nyeun Kim, Kook Hyun Kim}

Department of Internal Medicine, Yeungnam University Hospital, Daegu, Republic of Korea

\section{References}

1 Choi E, Byun JH, Park BJ et al. Duplication of the extrahepatic bile duct with anomalous union of the pancreaticobiliary ductal system revealed by MR cholangiopancreatography. Br J Radiol 2007; 80: e150 - 154

2 Gupta V, Chandra A. Duplication of the extrahepatic bile duct. Congenit Anom (Kyoto) 2012; 52: 176-178

\section{Bibliography}

DOI http://dx.doi.org/

10.1055/s-0034-1377216

Endoscopy 2014; 46: E316-E317

(c) Georg Thieme Verlag KG

Stuttgart · New York

ISSN 0013-726X

\section{Corresponding author \\ Kook Hyun Kim}

Division of Gastroenterology and Hepatology Department of Internal Medicine Yeungnam University College of Medicine 705-703, 317-1 Daemyung-dong Nam-gu

Republic of Korea

Fax: +82-53-6548386

kimkh@yu.ac.kr 